\title{
EMF-Aware Cell Selection in Heterogeneous Cellular Networks
}

\author{
Antonio De Domenico, Luis-Francisco Diez, Ramón Aguero, Dimitri Kténas, and Valentin Savin.
}

\begin{abstract}
The growing concern on the exposure of users to the Electromagnetic Field (EMF) has recently brought new challenges to the mobile research community. In this letter, we propose a novel cell association framework for heterogeneous cellular networks (HetNets), which aims to balance the load amongst heterogeneous cells so as to improve the resource usage and to increase the user satisfaction in terms of both data rate and EMF exposure. We model the cell selection problem as a General Assignment Problem (GAP) and we present two heuristic algorithms, which solve it with limited complexity. Our analysis shows that the proposed solutions lead to notable improvements with respect to legacy association schemes.
\end{abstract}

\section{INTRODUCTION}

Driven by the exponential increase of the mobile traffic, the wireless community has investigated solutions for enhancing the resource usage efficiency to improve the overall network performance. However, according to the latest European statistics [1], there is an increasing concern of the end-users about the potential health risks due to wireless communications.

The reduction of the EMF exposure poses additional challenges to the mobile industry: new methodologies, metrics, and architectures are required. Recently, to optimize network operations with respect to the EMF exposure, a new metric named as Exposure Index (EI) has been proposed [2]. The EI goes beyond state-of-the-art methodologies, by including statistical information and profiles. Moreover, one of the most specific features of the EI is that it does not only focus on the downlink (DL) exposure. In fact, although it is usually neglected, the uplink (UL) has a rather relevant impact on the overall exposure.

In the current cellular technology, a User Equipment (UE) selects the enhanced NodeB (eNB) that corresponds to the strongest Reference Signal Received Power (RSRP) [3]. Due to the power unbalance between small cell eNBs (SCeNBs) and Macro eNBs (MeNBs), this solution may prevent UEs from being served by the closest eNB. Hence, this increases the UL transmission power at the UEs, which in turns increases the user's exposure. Moreover, this approach limits the data rate, increases the UL interference, lowers the battery life, and

Antonio De Domenico, Dimitri Kténas, and Valentin Savin are with CEA, LETI, Minatec Campus, 17, rue des Martyrs 38054 Grenoble, France. Email: \{antonio.de-domenico, valentin.savin, and dimitri.ktenas\}@cea.fr.

Luis-Francisco Diez and Ramón Aguero are with the University of Cantabria, Communications Engineering Department, Santander, Cantabria, Spain. Email: \{ldiez, ramon\}@tlmat.unican.es.

This papers reports work undertaken in the context of the project LEXNET. LEXNET is a project supported by the European Commission in the 7th Framework Programme (Grant Agreement n. 318273). reduces the macro cell offloading. To deal with part of these problems, a Cell Range Expansion (CRE) can be used, where a positive bias is added to the strength of the small cell control signal. This approach, jointly with interference coordination schemes, which protect range expanded UEs from MeNB interference, results in improved fairness and capacity [4]. Nevertheless, some studies have shown that, when using large bias, too many UEs may be associated with the same SCeNB, leading to overload and interference issues [4]. In contrast, when small cells operate in a dedicated band, more aggressive CRE can be implemented due to the absence of the MeNB interference [5]. Recently, several works have investigated the cell association problem in HetNets, mainly for enhancing the network DL capacity [6].

Opposed to most of the existing works, we analyze the optimum cell association by jointly considering DL and UL communications, and we study the relationship between the EMF exposure and the users' Quality of Service (QoS). Besides, we present two user centric mechanisms that jointly reduce the EMF exposure induced by the UL and improve the user satisfaction in terms of the DL throughput goal; last, considering the system perspective, the proposed solutions distribute the load in the HetNet to enhance the (access/backhaul) network utilization efficiency.

\section{System MOdeL}

Following the recent investigations in 3GPP [7], our research focuses on HetNets where SCeNBs are densely deployed and operate in a dedicated carrier (see Figure 1).

We denote by $\mathcal{U}$ the set of UEs and by $\mathcal{B}$ the set of eNBs (which includes both MeNBs and SCeNBs) providing wireless services in the investigated HetNet. The average SINR between a user $i$ and an eNB $j$ can be modelled as

$$
\operatorname{SINR}_{i, j}=\frac{P_{j} \cdot \Gamma_{i j}}{I_{i, j}+\sigma^{2}}=\frac{P_{j} \cdot \Gamma_{i, j}}{\sum_{n \in \mathcal{B} \backslash j} P_{n} \cdot \Gamma_{i, n}+\sigma^{2}},
$$

where $P_{j}$ is the transmission power of the eNB $j$ and $I_{i, j}$ is the overall interference experienced by the UE $i$. Moreover,

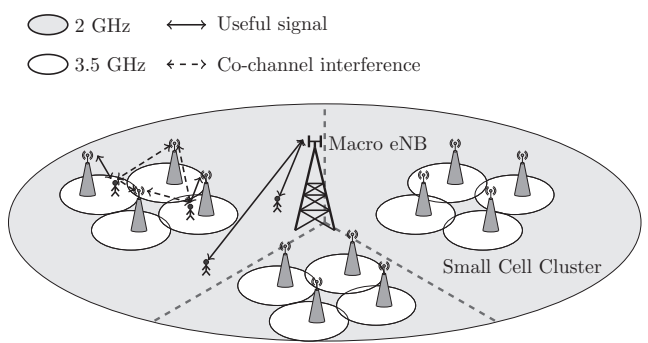

Figure 1: The heterogeneous network deployment under investigation. 
$\sigma^{2}$ is the additive noise power and $\Gamma_{i, j}$ is the channel gain (including path loss, shadowing, and antenna gain) between the UE $i$ and the eNB $j$. Note that the average SINR in (1) is due to measurements on the eNB control channels and it is independent of cell loads, fast fading, and resource allocation. We further denote

- the connectivity matrix $\mathbf{A}$, where $a_{i, j}$ equals 1 if a user $i$ is in the coverage area of eNB $j$ (i.e., $\operatorname{SINR}_{i, j}$ is larger than a given threshold) and 0 otherwise;

- a feasible assignment matrix $\mathbf{X}$, where $x_{i, j}$ is equal to 1 if user $i$ is served by eNB $j$ (0 otherwise), $\sum_{j \in \mathcal{B}} x_{i, j}=1$ $\forall i \in \mathcal{U}$, and $x_{i, j} \leq a_{i, j} \forall(i, j) \in \mathcal{U} \times \mathcal{B}$;

- the number of feasible assignment matrices $\mathrm{N}=$ $\prod_{i \in \mathcal{U}} \sum_{j \in \mathcal{B}} a_{i, j}$

- the set of all the possible service matrices $\mathcal{X}=$ $\left\{\mathbf{X}_{1}, \ldots, \mathbf{X}_{\mathrm{N}}\right\}$.

\section{A. DL data rate assessment}

For a given $\mathbf{X}$, the achievable data rate related to the link between $i$ and $j$ can be modelled as

$$
C_{i, j}=x_{i, j} \cdot B_{i, j} \cdot \eta_{i, j}
$$

where $B_{i, j}$ is the fraction of the band $B$ that the eNB $j$ allocates to the UE $i$ and $\eta_{i, j}=\log _{2}\left(1+\operatorname{SINR}_{i, j}\right)$ is the link spectral efficiency.

When the eNBs allocate more bandwidth to the UEs characterized by higher $\eta$, we have $B_{i, j}=\frac{k_{j} \cdot B \cdot \eta_{i, j}}{\sum_{y \in \mathcal{U}} x_{y, j} \cdot \eta_{y, j}}$, where $k_{j}=1$ if the backhaul does not limit the eNB capacity and $0<k_{j}<1$ otherwise. In the latter case, since $\sum_{y \in \mathcal{U}} x_{y, j} \cdot B_{y, j} \cdot \eta_{y, j}=C_{j}^{\mathrm{BH}}$, where $C_{j}^{\mathrm{BH}}$ is the backhaul capacity, we can find the value of $k_{j}=\frac{C_{j}^{\mathrm{BH}}}{B} \frac{\sum_{y \in \mathcal{U}} x_{y, j} \cdot \eta_{y, j}}{\sum_{y \in \mathcal{U}} x_{y, j} \cdot \eta_{y, j}^{2}}$, leading to

$$
B_{i, j}= \begin{cases}B \frac{\eta_{i, j}}{\sum_{y \in \mathcal{U}} x_{y, j} \cdot \eta_{y, j}}, & \text { if } B \frac{\sum_{y \in \mathcal{U}} x_{y, j} \cdot \eta_{y, j}^{2}}{\sum_{y \in \mathcal{U}} x_{y, j} \cdot \eta_{y, j}} \leq C_{j}^{\mathrm{BH}} \\ C_{j}^{\mathrm{BH}} \frac{\eta_{i, j}}{\sum_{y \in \mathcal{U}} x_{y, j} \cdot \eta_{y, j}^{2}}, & \text { otherwise }\end{cases}
$$

\section{B. EMF assessment}

To evaluate the EMF radiation in HetNets, we use a simplified version of the EI. The EI is able to model the exposure of different categories of people to different mobile technologies. However, here, we only focus on the UL of cellular networks, which is more relevant than the DL exposure due to the proximity of the device to the body; moreover, to simplify our analysis, we only consider adult users. With these assumptions, the user expected EI can be computed as the sum of the contributions due to different usages $u$ (i.e., data and voice services in indoor and outdoor scenarios) in the considered time periods $p$ (day and night) [2]

$$
E I_{i, j}=\sum_{p}^{N_{p}} \sum_{u}^{N_{u}} t_{p, u}^{\mathrm{UL}} \cdot \frac{S A R_{u}^{\mathrm{UL}}}{P_{\mathrm{TX}}^{\mathrm{ref}}} \cdot P_{i, j}^{\mathrm{UL}} \quad\left[\frac{\mathrm{mJ}}{\mathrm{Kg}}\right]
$$

Table I: Daily Statistics related to the Usage of Wireless Devices [2].

\begin{tabular}{|l|l|l|l|l|}
\hline \hline & \multicolumn{1}{|c|}{$\begin{array}{c}\text { Voice } \\
\text { Indoor }\end{array}$} & $\begin{array}{c}\text { Voice } \\
\text { Outdoor }\end{array}$ & $\begin{array}{c}\text { Data } \\
\text { Indoor }\end{array}$ & $\begin{array}{c}\text { Data } \\
\text { Outdoor }\end{array}$ \\
\hline$t_{p, u}^{\mathrm{UL}}(\mathrm{p}=$ day $)[s]$ & 242 & 242 & 1370 & 524 \\
\hline$t_{p, u}^{\mathrm{UL}}(\mathrm{p}=$ night $)[s]$ & 121 & 121 & 2335 & 306 \\
\hline$\frac{S A R_{u}^{\mathrm{UL}}}{P_{\mathrm{TX}}^{\text {ref }}}\left[\frac{1}{\mathrm{Kg}}\right]$ & $3.95 \mathrm{e}-06$ & $3.95 \mathrm{e}-06$ & $4.14 \mathrm{e}-06$ & $4.14 \mathrm{e}-06$ \\
\hline \hline
\end{tabular}

where $P_{i, j}^{\mathrm{UL}}$ is the power emitted by the UE $i$ to communicate with the serving eNB $j, t_{p, u}^{\mathrm{UL}}$ is the time spent in the usage $u$ during the time period $p$, and the ratio $\frac{S A R_{u}^{\mathrm{UL}}}{P_{\mathrm{TX}}^{\mathrm{ref}}}$ represents the whole body averaged specific absorption rate (SAR) that characterizes an adult during the usage $u$ and an incident reference power $P_{\mathrm{TX}}^{\text {ref }}$ (see Table I).

Finally, in 3GPP LTE, power control is used in the UL to mitigate interference and increase the device battery life. Accordingly, the power required by the UE $i$ to communicate with the eNB $j$ can be modelled as [8]

$$
P_{i, j}^{\mathrm{UL}}=\min \left\{P_{\max }, P_{0}+10 \log _{10} N_{\mathrm{RB}}^{\mathrm{UL}}+\lambda \cdot \frac{1}{\Gamma_{i, j}}\right\},
$$

where $P_{\max }$ is the maximum transmission power $(23 \mathrm{dBm})$ at the UE, $P_{0}$ is a UE-specific parameter $(-78 \mathrm{dBm}), N_{\mathrm{RB}}^{\mathrm{UL}}$ models the number of allotted resource blocks in the UL, and $\lambda$ is a path-loss compensation factor (0.8).

\section{Problem Statement}

In this work, we investigate whether it is possible to reduce the EMF exposure due to UL transmissions while increasing the number of UEs that meet their DL data rate target. On the one hand, due to the vicinity of the UE to the body, the EMF exposure is mainly determined by the UL; on the other hand, in current mobile technologies, the load is strongly asymmetric and enhancing the DL capacity is the main goal of operators. For a given service matrix $\mathbf{X}$, let's define the user satisfaction ratio $S(\mathbf{X})$ as the function that measures the fraction of UEs for which the DL capacity requirement $\left(C^{\mathrm{min}}\right)$ is met as

$$
S(\mathbf{X})=\frac{1}{|\mathcal{U}|} \cdot \sum_{i \in \mathcal{U}} \sum_{j \in \mathcal{B}} s_{i, j}
$$

where $s_{i, j}$ is a step function whose value is 1 if $C_{i, j} \geq C_{i}^{\text {min }}$ and 0 otherwise. Moreover,

$$
E I(\mathbf{X})=\sum_{i \in \mathcal{U}} \sum_{j \in \mathcal{B}} x_{i, j} \cdot E I_{i, j}
$$

is the aggregate EMF due to UL. Then, our optimization problem is given as follows

$$
\begin{aligned}
\mathbf{X}^{*} & =\underset{\mathbf{X}_{k} \in \mathcal{X}^{*}}{\operatorname{argmin}} E I\left(\mathbf{X}_{k}\right) \\
\text { where } \quad \mathcal{X}^{*} & =\left\{\mathbf{X}_{k} \in \mathcal{X} \mid S\left(\mathbf{X}_{k}\right)=\max _{\mathbf{X} \in \mathcal{X}} S(\mathbf{X})\right\}
\end{aligned}
$$

Note that (9) ensures that $\mathcal{X}^{*}$ contains all the service matrices that maximize (6).

Proposition: The above defined problem is NP-hard.

The GAP is a combinatorial problem in which each of $n$ tasks is optimally assigned to $m$ machines given the profit and the cost of each task as well as the resource available at each 
machine [9]. Accordingly, part of our assignment problem, i.e., finding the subset $\mathcal{X}^{*}$ can be mapped as a GAP, where

- the UEs and the eNBs are mapped to the tasks and to the machines, respectively;

- the user satisfaction $s_{i, j}$ and the data rate $C_{i, j}$ are mapped to the profit and cost of each task, respectively;

- the backhaul capacity $C^{\mathrm{BH}}$ is mapped to the resource constraint at each machine.

The GAP is known to be NP-hard while deciding if a feasible solution exists is NP-complete; therefore, the overall described assignment problem is NP-hard.

\section{Proposed Solution}

In this section, we propose two centralized algorithms (named as Max Sat. and EMF-Aware) to deal with the EMFAware cell selection problem presented in Section III. These schemes require coordination amongst eNBs: a distributed approach is feasible but it increases the required overhead. A practical implementation is to find a solution at the MeNB by gathering information from the nearby SCeNBs. Note that the proposed process can be seen as a self-organizing network (SON) functionality that does not require fast adaptation to e.g., mobility and fast fading [10]; in fact, SINR reporting can be exchanged on a large time scale (i.e., seconds), which limits the overhead and the latency requirements.

The proposed algorithms start from a given solution of the cell selection problem (e.g., based on the RSRP), and iteratively evolve towards a more beneficial association. At each iteration, they evaluate every possible single change in the current association (first step) and then select the more beneficial change (second step). The algorithms stop after a limited number of iterations, when the achievable gain becomes lower than a small non-negative value $\epsilon$.

Let $\mathbf{X}_{n}$ be the user assignment that maximizes $P_{j} \cdot \Gamma_{i j} \forall$ $(i, j) \in \mathcal{U} \times \mathcal{B}$,

(1) First Step: Initialization

- Calculate $E I\left(\mathbf{X}_{n}\right)$ and $S\left(\mathbf{X}_{n}\right)$;

- For all $(i, j)$, s.t. $a_{i, j}=1$, compute (6) and (7) if we change $\mathbf{X}_{n}$ by associating (respectively, deassociating) the user $i$ to (respectively, from) $j$; then, compute the gains $\Delta_{\mathrm{S}}$ and $\Delta_{\mathrm{EI}}$ with respect to the reference association, due to the possible reassignments.

(2) Second Step: One-user reassignment

- IF Max Sat., Find the set $\mathcal{X}^{*}$ that maximizes $\Delta_{\mathrm{S}}$;

- ELSE IF EMF-Aware, Find the assignment set for which $\Delta_{\mathrm{EI}} \geq 0$; then find its subset $\mathcal{X}^{*}$ that maximizes $\Delta_{S}$;

- IF $\max \Delta_{\mathrm{S}} \leq \epsilon$, exit (the algorithm outputs the current user assignment);

- ELSE find $\mathbf{X}_{k} \in \mathcal{X}^{*}$ that maximizes $\Delta_{\mathrm{EI}}$ and update the user assignment, accordingly.

- Set $\mathbf{X}_{n}=\mathbf{X}_{k}$, then go to step (1).

Proposition: In the proposed solutions the number of satisfied users is improved at each new iteration. Hence, the algorithms converge when a further improvement is not possible by a new reassignment of one single user.

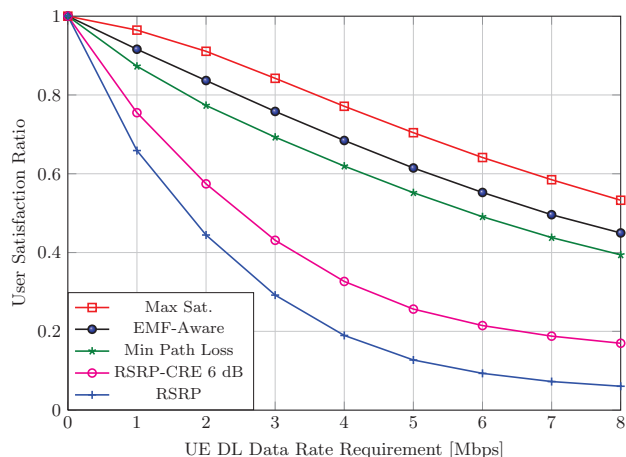

Figure 2: User satisfaction ratio Vs. DL data rate requirement.

\section{Simulation Results}

In this section, we assess the effectiveness of the proposed solutions, which attempt to limit the EI while considering the side constraint of maximizing the user satisfaction. We compare their performance with respect to the approach where each UE is served by the eNB associated with the strongest RSRP and the scheme where each UE is associated with the closest eNB (Min Path Loss). We also consider a CRE bias of $6 \mathrm{~dB}$ to increase the macro cell offloading.

Our evaluation scenario is composed by a tri-sectorial macro cell (3 MeNBs) and 60 UEs. Moreover, three small cell hotspots, each one composed by 4 neighbouring SCeNBs (see Figure 1), are deployed in the macro cell. The eNBs are characterized by a $C^{\mathrm{BH}}$ of $40 \mathrm{Mbps} .80 \%$ of the UEs are indoor, $2 / 3$ of them are located in the small cell hotspots, and remaining UEs are uniformly distributed in the macro cell. Other relevant parameters follow 3GPP TR 36.872 [7]. The results are averaged over $10^{3}$ independent runs. At the beginning of each run, the clusters of SCeNBs and the UEs are randomly deployed in the macrocell area. Finally, we consider a stopping parameter $\epsilon=10^{-6}$.

Figure 2 shows the user satisfaction ratio with respect to the DL requirements. Note that $S(\mathbf{X})$ can be seen as the DL rate complementary cumulative distribution function. By implementing the classic RSRP scheme, most of the UEs are associated with the MeNBs, which limits the resources allotted to the UEs with poor SINR and achieves the worst performance. CRE enhances the user satisfaction by offloading UEs from overloaded MeNBs to lightly loaded SCeNBs. Implementing CRE is particularly beneficial in the region with high rate requirements, where it achieves $2.8 \mathrm{X}$ the user satisfaction of the RSRP scheme. By associating the UEs to the closest eNBs, the system does not suffer from the power unbalance between SCeNBs and MeNBs, which enables to effectively share the network load and to achieve up to $6.5 \mathrm{X}$ the performance of the RSRP solution. It is worth to recall that, in a co-channel deployment, the Min Path Loss scheme might lead to limited performance due to the strong macro cell interference [4]. The proposed schemes further enhance the user satisfaction through an optimized load balancing that associates UEs characterized by poor SINR to eNBs with large resource availability and UEs with high SINR to loaded eNBs. Accordingly, UEs at the cell edge may meet the data rate requirements at the cost of lower throughput experienced by UEs located nearby the eNBs. As expected, the Max Sat. 


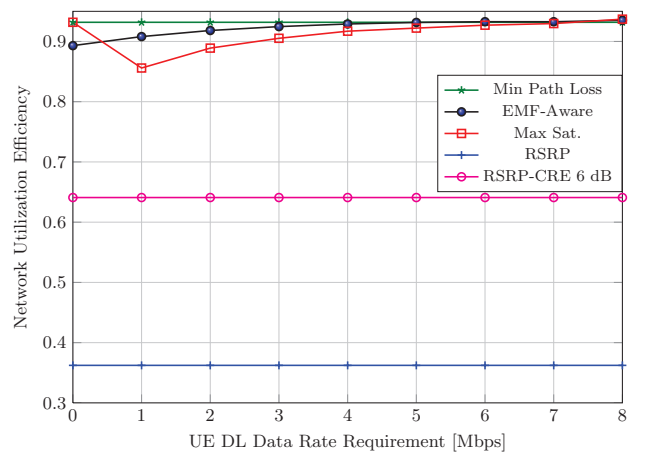

Figure 3: Cell Resource Utilization Vs. DL data rate requirement.

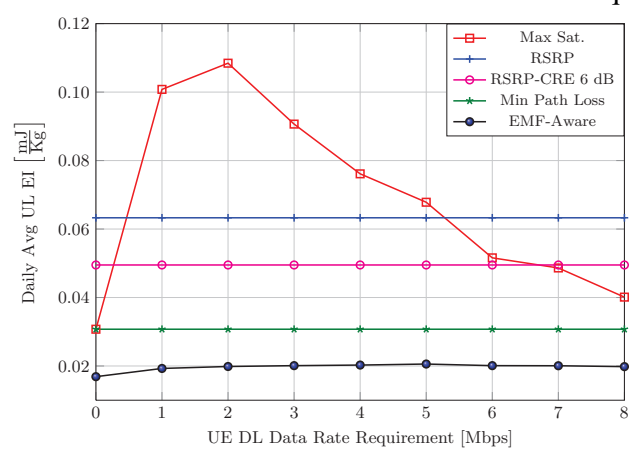

Figure 4: UL Exposure Index Vs. DL data rate requirement.

outperforms the EMF-Aware approach since the latter avoids those associations leading to high EI. The Max Sat. and the EMF-Aware yield up to $8.8 \mathrm{X}$ and $7.4 \mathrm{X}$ the user satisfaction of the reference scheme.

Figure 3 shows the network utilization efficiency (the ratio between the achieved HetNet capacity and the aggregate backhaul capacity) related to the different approaches. When using the RSRP scheme, most of the UEs are served by the MeNB and a high number of small cells are idle, which leads to the worst performance. CRE enables to increase the usage of SCeNB resources through the macrocell offloading; however, Max Sat., EMF-Aware, and the Min Path Loss achieve up to 2.6X the resource utilization of the RSRP solution. Although these three algorithms are characterized by similar resource utilization, the proposed solutions, which fairly distribute resources to increase the user satisfaction, are characterized by slightly lower performance than the Min Path Loss, which blindly allocates resources to the UEs with larger SINR.

Figure 4 shows the daily EI due to UL with respect to the $\mathrm{DL}$ rate requirement. In this simulations, $N_{\mathrm{RB}}^{\mathrm{UL}}$ in (5) is computed such that each UE achieves $1 \mathrm{Mbit} / \mathrm{s}$ in the UL. As expected, associating UEs to nearby eNBs limits the required UL power per resource block; accordingly, CRE and Min Path Loss scheme are beneficial in terms of exposure with respect to the reference solution based on the RSRP. However, better performance can be achieved through the EMF-Aware solution, where the load balancing reduces the required $N_{\mathrm{RB}}^{\mathrm{UL}}$ (e.g., by offloading to the MeNB the small cell UEs characterized by high uplink interference). On the other hand, by inspecting Figures 2 and 3, we can state that to further increase $S(\mathbf{X})$, it is necessary to implement cell selection patterns that increase the EI. In fact, the Max Sat. results in the highest $\mathrm{EI}$ in the range of low-medium rate requirements, where it is possible to satisfy most of the UEs. On the contrary,
Table II: Running Time of Max Sat. Vs. Number of Users $\left(\mathbf{C}_{\min }=\right.$ $2 \cdot 10^{6}$ Mbps; $|\mathcal{B}|=15$ ).

\begin{tabular}{|c|c|c|c|c|c|c|}
\hline \hline$|\mathcal{U}|$ & 9 & 15 & 30 & 45 & 60 & 90 \\
\hline Average Iterations & 3.4 & 5.7 & 11.5 & 14.7 & 16 & 21.3 \\
\hline Average Time [ms] & 7.6 & 19 & 79 & 169 & 276 & 640 \\
\hline \hline
\end{tabular}

in the high rate requirements region, only few UEs can meet the rate target and the average EI can be strongly reduced.

Finally, to assess the complexity of the proposed schemes, we evaluate the average number of iterations and the associated running time. To obtain these results, we have used an Intel i5 3.2 GHz, equipped with 4 GB RAM. Table II shows the results for the Max Sat. scheme, which is more time consuming than the EMF-Aware; the results show that it has a linear dependency on the number of UEs. Moreover, they confirm that the algorithm converges in a time range satisfying the requirements of $3 \mathrm{GPP}$ load balancing functions [10].

\section{CONCLUSION}

Most of the current cell selection schemes for HetNets are based only on the radio link quality, which limits the macro cell offloading, leading to congestion situations at cells with capacity-limited backhaul, and increases the required UL power. To solve this problem, we have proposed two schemes that enhance the user satisfaction in terms of both the DL data rate and the EMF exposure; furthermore, they increase at the same time the utilization efficiency of the network resources. Our analysis underlines that, to improve the HetNet performance through load balancing, it is necessary to simultaneously take into account the users' requirements, the cell load and signal strength, the interference level, and the backhaul capacity. Moreover, our results show that satisfying the data rate requirements of UEs with poor SINR comes at the cost of increasing the average EMF exposure. To appropriately deal with this trade-off, in our future work, we will integrate dual connectivity schemes, where DL and UL traffics are served by distinct eNBs, and we will pose problem formulations to identify solutions with guaranteed performances.

\section{REFERENCES}

[1] TNS Opinion \& Social, "Eurobarometer 73.3 - Electromagnetic Fields," June 2010.

[2] LEXNET, "D2.4 Global Exposure Metric Definition," November 2013.

[3] S. Parkvall, et al., "Heterogeneous network deployments in LTE," Ericsson Review, vol. 2, 2011.

[4] D. Lopez-Perez, X. Chu, and I. Guvenc, "On the Expanded Region of Picocells in Heterogeneous Networks," IEEE J. Sel. Top. Sign. Proces., vol. 6, no. 3, pp. 281-294, 2012.

[5] J. Andrews, et al., "An overview of load balancing in hetnets: old myths and open problems," IEEE Wireless Communications, vol. 21, no. 2, pp. 18-25, April 2014.

[6] Q. Ye, et al., "User Association for Load Balancing in Heterogeneous Cellular Networks," IEEE Trans. on Wireless Commun., vol. 12, no. 6, pp. 2706-2716, 2013.

[7] 3GPP, "TR 36.872, Small cell enhancements for E-UTRA and EUTRAN-Physical layer aspects (Release 12)," V12.1, December 2013.

[8] C. Castellanos, et al., "Performance of Uplink Fractional Power Control in UTRAN LTE," in IEEE VTC Spring, May 2008, pp. 2517-2521.

[9] S. Martello and P. Toth, Knapsack problems. Wiley New York, 1990.

[10] M. Nohrborg, "Self-Organizing Networks," http://www.3gpp.org/ technologies/keywords-acronyms/105-son, last access July 2014. 